\title{
Philosophiques
}

\section{Pour réhabiliter la notion d'incorporation des entités culturelles : réponse à Dale Jacquette}

Joseph Margolis

Volume 13, numéro 2, automne 1986

URI : https://id.erudit.org/iderudit/203323ar

DOI : https://doi.org/10.7202/203323ar

Aller au sommaire du numéro

Éditeur(s)

Société de philosophie du Québec

ISSN

0316-2923 (imprimé)

1492-1391 (numérique)

Découvrir la revue

Citer ce document

Margolis, J. (1986). Pour réhabiliter la notion d'incorporation des entités culturelles : réponse à Dale Jacquette. Philosophiques, 13(2), 333-343.

https://doi.org/10.7202/203323ar d'utilisation que vous pouvez consulter en ligne.

https://apropos.erudit.org/fr/usagers/politique-dutilisation/ 


\title{
INTERVENTIONS
}

\section{POUR RÉHABILITER LA NOTION D'INCORPORATION DES ENTITÉS CULTURELLES : RÉPONSE À DALE JACQUETTE}

\author{
par Joseph Margolis*
}

L'article de Dale Jacquette, «Émergence et incorporation d'après Margolis » (cf. Philosophiques, Vol. XIII, nº 1, pp. 53-63) me fournit une bonne occasion de clarifier, afin de dissiper divers malentendus possibles, les grandes lignes de mon approche concernant l'ontologie des entités culturelles - en particulier, les œuvres d'art, les personnes, les mots, les énoncés, et d'autres entités du même genre. Je ne traiterai ici que de la nature et de certains détails de ma thèse. Les arguments dialectiques qui la favorisent, par opposition à d'autres options, comme le matérialisme réducteur (reductive materialism), le dualisme cartésien ou l'idéalisme, ont déjà été présentés ailleurs et exigeraient, de toute manière, une discussion théorique beaucoup trop étendue.

Mon présupposé fondamental comporte trois volets:

1) Les entités culturelles ne sont pas identiques aux entités purement physiques, ni analysables en des termes qui

* Note de la rédaction. La version anglaise de l'étude de Dale Jacquette a été publiée dans le Journal of Aesthetics and Art Criticism après avoir paru dans Philosophiques. Quant à la réponse de Joseph Margolis, elle paraît d'abord dans le Journal of Aesthetics and Art Criticism.

La traduction du texte de Joseph Margolis a été effectuée par André Leclerc et a été révisée par Claude Savary. 
sont réductibles à ceux qui sont adéquats pour l'identification, la description et l'explication des entités purement physiques ;

2) Les entités culturelles ne sont pas vraiment séparables (bien qu'elles soient numériquement distinctes) des entités purement physiques, et ne sont pas identifiables sans qu'on tienne compte des propriétés des entités purement physiques ;

3) Il y a des propriétés qui distinguent les entités culturelles des entités non-culturelles, génériquement et uniformément pour toutes les espèces caractéristiques d'entités culturelles, en tant que conditions ontiques nécessaires et suffisantes.

Je me permets d'ajouter que je ne conçois pas.la clause 3 d'une manière essentialiste. Il se peut que l'on doive reconnaître l'existence de cas limites; la clause 3 constitue en fait, en tant que présupposition, une recommandation concernant les propriétés qui définissent les entités culturelles, là où une notion relativement faible de définition peut être autorisée - par exemple, comme caractérisant fortement des exemplaires normalement reconnus d'une manière qui soit compatible avec une certaine tolérance conceptuelle pour les cas difficiles qui tombent sous l'extension recommandée.

Même si ces trois clauses sont ici traitées comme des présuppositions pour les fins que je poursuis dans cette réponse à Jacquette, chacune d'entre elles, j'y insiste, peut être présentée à partir de fondements indépendants, comme une thèse bien établie. J'ai toujours appelé les théories qui satisfont les conditions 1-3 les matérialismes non-réducteurs (non-reductive materialisms). Mon intérêt premier est d'arriver à démontrer qu'une ontologie adéquate pour les entités culturelles n'est réalisable que sous la forme d'un matérialisme non-réducteur. Mais le champ des entités culturelles ne couvre pas toute l'étendue du matérialisme non-réducteur, parce qu'il est tout à fait possible que ce dernier, sans tenir compte de sa portée pour une ontologie de la culture, puisse rendre compte de certaines différences entre les phénomènes physiques et biologiques, ou entre les phénomènes psychiques et physiques, ou encore d'autres différences du même 
genre. Par suite, la simple défense d'un matérialisme nonréducteur ne fournit pas forcément une solution portant sur le problème de l'ontologie des entités culturelles - ou l'ontologie des phénomènes biologiques. C'est sur le problème plus étroit de l'ontologie du culturel que je voudrais insister ici.

Néanmoins, en tenant compte seulement des considérations qui précèdent, il est surprenant de constater à quel point le contre-exemple de Jacquette est défectueux et manque même de pertinence. Je ne veux pas dire que ses contre-exemples ne sont pas théoriquement intéressants ou même importants. Mais le moindre coup d'œil montrera que son prétendu contre-exemple impliquant une montagne et une vallée n'implique tout simplement pas d'entités culturelles, si on considère n'importe quel critère raisonnable déterminant ce qui compte comme cuvres d'art, personnes, mots, énoncés, actions humaines, récits (bistories), institutions, pratiques, etc. Jacquette ne semble pas ignorer que la définition de ce que j'appelle incorporation (embodiment) est la définition d'une propriété ontique qui définit les entités culturelles - et certainement pas une propriété définissant le matérialisme non-réducteur. C'est bien sûr en partie pour cette raison que l'on doit distinguer l'ontologie des phénomènes biologiques de celle des phénomènes culturels, les deux pouvant être traités dans le cadre d'un matérialisme non-réducteur. Malgré tout, pour mettre à l'épreuve ma définition de l'incorporation, Jacquette introduit spécifiquement un exemple de quelque chose qui ne peut, d'aucune façon, compter comme phénomène culturel.

Je peux affirmer que ce n'était pas mon intention, dans Persons and Minds, d'éviter la redondance en définissant l'« incorporation»; il me semblait qu'il y avait quelque avantage à être clair à propos d'une distinction technique et relativement peu familière; aussi me fait-il plaisir d'admettre que mes conditions (i)-(vii) peuvent ne pas être aussi économiques qu'on pourrait le désirer. Mais ces conditions ne sont satisfaites, de toute manière, que par des entités culturelles. Ma solution au problème de l'ontologie de la culture - en accord avec les présupposés 1-3 donnés plus haut -, est que toutes les entités culturelles et seulement ces entités exhibent conjointement les propriétés que j'appelle incorporation et être une-occurrence-d'untype (being a token-of-a-type). La suggestion que fait Jacquette 
d'une condition (vi') montre clairement qu'il comprend mal le but de ma formulation de (vi), qui est logiquement méticuleuse, et qu'il interprète dans la ligne d'une mécompréhension des relations entre ces deux conditions ontiquement nécessaires et suffisantes. Ma solution implique, par exemple, qu'une gravure à l'eau-forte de Dürer peut être reproduite en plusieurs exemplaires par diverses impressions, et le même mot particulier peut être énoncé dans des séquences de sons tout à fait différentes; mais la gravure et le mot réels ne peuvent devenir effectifs qu'en ayant une occurrence dans une certaine impression ou énonciation. (Certains problèmes concernant les plaques servant à l'impression des gravures, les partitions musicales, etc., compliquent quelque peu mais ne détruisent pas la distinction entre une occurrence et son type). C'est donc un trait révélateur du contre-exemple de Jacquette qu'il ne considère jamais, relativement à ses prétendus exemples d'entités culturelles, la propriété : être une-occurrenced'un-type.

Mais une fois qu'il est clair que l'incorporation (n'insistons que sur cette propriété puisque Jacquette ne discute pas de l'autre qui vient d'être mentionnée) n'est proposée qu'en tant que propriété définissant les entités culturelles, n'importe quel prétendu contre-exemple devrait alors exhiber ces sortes de propriétés qui sont caractéristiques des entités culturelles, et pas seulement des propriétés de quelque espèce que ce soit qui diffèrent des sortes de propriétés que d'autres entités exhibent de fait. Ainsi, le simple fait que les vallées et les montagnes ne peuvent avoir en commun exactement les mêmes sortes de propriétés (la cinquième prémisse de Jacquette) manque tout à fait de pertinence pour mettre à l'épreuve le concept d'incorporation - quelque soit par ailleurs l'intérêt qu'il y a à examiner le sens de cette relation entre les montagnes et les vallées. En conséquence, la conclusion de Jacquette que «La définition implique que la vallée $v$ est matériellement incorporée dans la montagne $m »$, est fausse, parce qu'il emploie incorrectement le terme « incorporation» dans un contexte d'où il est expressément exclu. (D'ailleurs il serait plus vraisemblable de dire qu'une "vallée» doit être «incorporée» dans quelque chose de "valonneux», et non dans quelque chose de montagneux). Je pense que Jacquette confond tout simplement la question du matérialisme 
non-réducteur avec celle de l'incorporation. Je l'ai dit plus haut, l'incorporation est une propriété «essentielle» des entités culturelles - et toutes les entités qu'elles caractérisent relèvent par le fait même du matérialisme non-réducteur. Jacquette me semble avoir manœuvré de façon à détacher les conditions (i)-(vii) (de ma définition) de leur référence exclusive à des entités culturelles. C'est du moins ce qu'il semble faire pendant un moment, mais comme il mentionne, un peu plus loin, les œuvres d'art «trouvées» (found art), les œuvres toutes faites (readymades) et l'intentionnalité, il devient difficile de croire qu'il ignorait, en avançant son soi-disant contre-exemple (à l'aide de ses conditions (1)-(7)), qu'il utilisait incorrectement la notion d'incorporation. (J'admets qu'il est difficile de croire qu'il était conscient de ce qu'il faisait).

Jacquette dit explicitement, dans le contexte même où il offre son contre-exemple, que «Margolis n'affirme pas que toutes les entités matériellement incorporées sont culturellement émergentes...». Cependant, à la page 22 de mon ouvrage $A r t$ and Philosophy (1980), tout de suite après avoir donné la liste des conditions (i)-(vii) (qui reprend la liste que Jacquette cite à partir de Persons and Minds (1978)), j'écris : "Le "est" de l'incorporation est invoqué dans des contextes culturels pour faciliter notre discours à propos des particuliers culturels»; «... le concept d'incorporation permettra, sans présupposés dualistes, de rendre plus facile une explication non-réductionniste du rapport entre la nature physique et la culture humaine». Et à la page 13 de Culture and Cultural Entities (1984), je dis : "Les entités culturelles sont incorporées et sont des occurrences-de-types. Je crois que toutes les entités culturelles et seulement ces entités exhibent ces deux caractéristiques... [L'incorporation] est un rapport sui generis qui vaut seulement dans le cas des entités culturelles et qui peut aussi rendre compte de l'irréductibilité de l'Intentionnel...». Ainsi, Jacquette s'est tout simplement trompé en soutenant que je "n'affirme pas que toutes les entités matériellement incorporées sont culturellement émergentes».

Je reconnais qu'en écrivant Persons and Minds, j'ai été attiré, si je puis dire, par des problèmes impliquant l'émergence biologique (par exemple, le rapport abeille/ruche), et par des problèmes concernant les propriétés en tant qu'opposées aux 
entités; mais je crois ne jamais offrir d'exemples d'incorporation qui ne sont pas des exemples de phénomènes culturellement émergents. A la page 234 de Persons and Minds, d'ailleurs citée par Jacquette, $j$ 'identifie l'incorporation comme une propriété de ces choses qui peuvent être des occurrences-de-types, et je dis alors expressément que seules les entités culturellement émergentes peuvent être des occurrences-de-types. Mais je concède que dans cet ouvrage (l'un de mes premiers sur la question), je parle d'incorporation comme d'« une relation qui vaut au moins [sic] entre les corps physiques et les entités qui n'existent que dans un environnement (setting) culturel, ces dernières ne pouvant vraiment exister que via un corps physique de quelque espèce que ce soit». J'expose alors faiblement (au sens logique) mon point de vue, mais je n'étends jamais l'usage du terme - là ou ailleurs aux entités non-culturelles; et de même, dans Art and Philosopby et Culture and Cultural Entities, et partout ailleurs où je m'occupe de la question, j'utilise toujours la formulation forte. Dans Persons and Minds, $\mathrm{j}$ 'introduis la notion d' incorporation" juste après avoir fait les remarques citées plus haut. Tout ceci ne

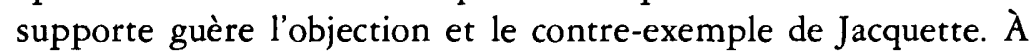
mon avis, ce que Jacquette veut dire lorsqu'il parle du caractère «matériellement incorporé» de sa vallée dans une montagne ou un paysage environnant, c'est simplement qu'elle est «matériellement en continuité avec» la montagne ou le paysage, ou encore qu'elle « recouvre matériellement» et partiellement la montagne ou le paysage environnant. (C'est là sûrement ce que veut dire partager un « versant» ou une pente). Mais le but que je poursuis en parlant de l'incorporation est de bien faire voir ce qui suit : il y a une différence parmi les propriétés, une différence parmi les sortes de propriétés, une différence parmi les sortes de propriétés qui implique l'émergence, une différence impliquant qu'il y a émergence et qui implique l'émergence culturelle, et par suite une différence qui a pour conséquence des sortes de propriétés qui n'apparaissent qu'au niveau des phénomènes culturellement émergents. Rien de tout cela ne peut être saisi par les notions de recouvrement et de continuité matériels, ou par n'importe quel type de relation similaire pouvant tenir compte de l'exemple de Jacquette d'une vallée «flanquée» d'une montagne et s' « enroulant» autour d'elle. 
Parce qu'il juge défectueuse ma définition de l'incorporation, Jacquette considère ensuite la possibilité de l'améliorer. Mais les possibilités qu'il suggère mentionnent à peine les propriétés qui distinguent les entités culturelles comme telles - je veux dire: Jacquette tente vraiment de faire passer pour une amélioration substantielle exactement le genre de considérations qu'on peut trouver, dès le début, dans ma propre tentative en vue de construire la notion d'incorporation comme s'appliquant - à titre d'analyse - d'abord et avant tout aux entités culturelles! Le fait est que mes conditions (i)-(vii) font et furent présentées comme des propriétés ontologiques tout à fait formelles, et ne furent jamais destinées à servir de traits distinctifs de différentes sortes ou espèces de choses en général; ces conditions concernent plutôt seulement les choses qui sont déjà perçues, pré-théoriquement pour ainsi dire, comme spécimens culturels. Par ailleurs, il est parfaitement clair que les conditions (i)-(vii) ne font aucunement mention d'entités culturelles: elles sont abstraites par la réflexion sur les entités culturelles et sont délibérément formulées de façon à marquer ce qui est formellement distinctif de ces entités. En conséquence, les conditions (iii)-(v) ne nous invitent pas du tout à introduire n'importe quelles propriétés arbitrairement sélectionnées que possèderaient différents particuliers, mais à considérer seulement ces propriétés qui distinguent correctement les entités culturelles des entités non-culturelles. Les conditions (i)-(vii) ne sont pas, et ne pourraient être destinées à marquer les traits originaux permettant d'identifier les entités culturelles: elles valent seulement des entités déjà reconnues comme culturelles; ce qui signifie que pour les entités ayant les propriétés qui sont habituellement reconnues comme culturellement pertinentes (comme celles des œuvres d'art, personnes, mots, énoncés, etc.), on constatera que toutes ces entités et seulement de telles entités ont les propriétés ontiques distinctives d'être incorporé et d'être des-occurrences-de-types.

Une autre façon de présenter les choses est de montrer que les entités culturelles se distinguent en tant qu'elles sont complexes et émergentes, et peuvent se manifester en plusieurs occurrences, en dépit du fait que ce sont des choses particulières. Je trouve que c'est là un fait des plus remarquables - et il me semble que Jacquette ne s'y intéresse pas le moins du monde. La 
clause 1 de mon présupposé fondamental présenté au tout début était conçue comme devant accommoder à la fois l'émergence des entités culturelles et le fait qu'elles sont des occurrences-de-types; ces deux traits sont incompatibles avec le matérialisme réducteur. La clause 2 était destinée à accommoder à la fois la complexité des entités culturelles à leur niveau d'émergence et leur relation essentiellement non-disjonctive avec le monde physique; ces deux caractéristiques sont incompatibles avec le dualisme et l'idéalisme. La clause 3 devait tenir compte des propriétés sui generis de l'incorporation et de celle qui consiste à être une occurrence-d'un-type - que seules les entités culturelles satisfont, c'est-à-dire les entités satisfaisant les clauses 1 et 2 ; la clause 3 , en conséquence, devait rassembler, mais sans encore les mentionner, des propriétés distinctives quelconques qui sont localement assignables aux entités normalement reconnues comme culturelles.

Ainsi, la thèse quelque peu provocante que j'ai offerte régulièrement à mes lecteurs peut être formulée comme suit: les entités culturelles (œuvres d'art, personnes, mots, énoncés, etc.) : (a) sont des particuliers; (b) sont émergentes (c'est-à-dire, nonréductibles en termes physicalistes); (c) sont complexes (c'est-àdire, ne sont pas détachables ou indépendants des particuliers physiques); (d) sont incorporées (c'est-à-dire, sont telles qu'elles partagent des propriétés physiques avec leurs particuliers incorporants, mais possèdent en plus des propriétés qui n'apparaissent qu'au niveau de leur émergence); (e) sont des occurrences-detypes (c'est-à-dire, sont exemplifiées par plusieurs particuliers qui comptent, sous une règle appropriée, comme exemples (instances) du même particulier - comme les reproductions de la même gravure ou les interprétations de la même sonate). Même si les clauses (a)-(e) fournissent un ensemble de conditions nécessaires et suffisantes pour être une entité culturelle, les caractéristiques mentionnées sont purement formelles et doivent être abstraites eu égard à des propriétés qui s'avèrent être des propriétés locales de sortes ou espèces de choses - par exemple, être linguistique, historique, intentionnel, délibéré, responsable, libre, moral, artistique, politique, etc. - , qui justifient et supportent l'abstraction. Rien, dans ce cas, n'apparaît simplement comme incorporé ou comme occurrence-d'un-type. Jacquette, il me semble, a tout à fait raté ce point. 
Ces considérations montrent bien la faiblesse du contreargument de Jacquette, qui rend apparemment triviale ma position concernant les haeccéités. Lorsque je fais remarquer - condition (v) - que les particuliers incorporés ont des propriétés d'une espèce que ne peuvent posséder les particuliers incorporants, il est clair que je ne veux pas dire quelques propriétés (prises au hasard) que l'un peut posséder et l'autre pas (dans un ensemble de particuliers pris au hasard), mais plutôt quelques propriétés (que l'un peut posséder mais pas l'autre) qui distinguent normalement des entités culturelles telles que les ouvres d'art, les personnes, les mots, les énoncés, etc. Invariablement, Jacquette favorise la première lecture, laquelle diminue vraiment la pertinence de son objection. Il suggère alors d'introduire des propriétés intentionnelles. Mais j'avais naturellement à l'esprit de telles propriétés intentionnelles, et j'en ai fait grand cas dans les livres auxquels Jacquette réfère; cependant, à mon avis du moins, nous manquons encore d'une explication satisfaisante de ces propriétés intentionnelles qui sont proéminentes et distinctives des phénomènes culturels. Je n'insisterai pas ici sur ce point, sinon pour dire que, selon moi, certaines propriétés intentionnelles (même chez les animaux) peuvent être assignées à des entités situées «en-dessous» du niveau de l'existence culturelle. Jacquette reconnaît, sans toutefois y accorder trop d'importance, que j'introduis des propriétés intentionnelles. Et là il sent le besoin d'être plus circonspect, mais ne fait guère plus que de remarquer que «Rien ne nous garantit que l'intentionnalité est la seule propriété irréductible susceptible de distinguer les particuliers émergents de leurs incorporations matérielles ». Fort bien. Mais cela montre clairement que Jacquette savait bien que les conditions (i)-(vii) doivent être appliquées à des entités qui de fait exhibent, en un certain sens restreint acceptable, des propriétés comme l'intentionnalité et d'autres du même genre; et cela va à l'encontre de toute son argumentation.

Le reste de l'intervention de Jacquette se concentre sur l'apparente facilité avec laquelle l'inclusion de l'intentionnalité (ou de quelque autre propriété exclusive des entités culturelles) - inclusion devant améliorer la définition de l'incorporation peut être annulée par simple référence aux œuvres d'art «trouvées» (found art) et aux cuvres toutes faites (readymades). La 
seule chose que je puis dire ici c'est que Jacquette a mal lu mon explication concernant de telles œuvres d'art. Avant tout, j'admets - j'y insiste - que ces cas sont problématiques et qu'ils ne représentent pas des cas vraiment centraux (quoique d'une certaine importance). Deuxièmement, je donne effectivement une solution à l'apparente énigme de ces ouvres d'art, une solution consistante avec ma thèse (voir, par exemple, Art and Philosophy, chap. 2 - où la notion d'incorporation est introduite dans ce livre). Précisément, ma solution est que les œuvres d'art trouvées, les œuvres d'art toutes faites et autres œuvres du même genre, ne sont pas simplement des objets physiques élevés à la dignité d'œuvre d'art. Jacquette écrit: "Le rôle de l'artiste ici ne consiste pas tant à construire matériellement qu'à attirer l'attention sur ces objets et à les étiqueter comme objets d'art» (je souligne). Non, l'artiste crée effectivement les œuvres trouvées et toutes faites, bien qu'il ne produise ou ne construise pas l'objet physique ou l'artefact qui incorpore l'œuvre d'art créée: je dis explicitement, à propos de Duchamp par exemple, qu'il a fait le Porte-bouteilles, et qu'il n'a pas fait un porte-bouteilles (p. 20). Ainsi, il est vraiment tout à fait absurde que Jacquette affirme (selon ma position ou en général) qu'un certain quelque chose de physique, une vallée $v$, puisse avoir la propriété culturellement pertinente d'intentionnalité ; ou encore, qu'un exemple d'œuvre d'art trouvée, la Vallée $V$ («produite» simplement en la nommant telle) puisse avoir une propriété intentionnelle simplement en rattachant cette propriété à la vallée $v$. Si mon point de vue est viable (et l'on peut penser ici à Christo), la Vallée $V$ sera probablement incorporée dans la vallée $v$. Il n'y a absolument aucune raison suivant laquelle la relation entre les objets physiques incorporants et les objets culturels incorporés devrait être isomorphe (pour autant que l'analyse et l'explication sont concernés) avec la relation entre les objets qui sont contenus matériellement dans d'autres objets ou qui, en partie, se recouvrent l'un l'autre matériellement. Il en est ainsi simplement parce que, selon ma condition (vii), les particuliers incorporants ne sont pas des parties propres des particuliers incorporés. Par suite, quelque soit la difficulté qu'il y a à démêler les montagnes des vallées, cela n'a absolument rien à voir avec les cuvres d'art dont la création occasionne l'individuation de leurs particuliers incorporants - ce 
qui vaut aussi des œuvres trouvées ou toutes faites. Par exemple, on peut facilement imaginer que la Vallée $V$ (l'œuvre d'art trouvée de Jacquette) est incorporée dans une certaine étendue de paysage physique (qui peut inclure plusieurs montagnes et qui sait quoi encore)! Ainsi, il est tout à fait possible que la Vallée $V$ puisse avoir des propriétés physiques qu'une certaine montagne $m$ (disons, une partie de ce dans quoi la Vallée $V$ est incorporée) ne possède pas et ne pourrait posséder. C.Q.F.D. La condition (viii) de Jacquette - une autre de ses améliorations potentielles - non seulement passe entièrement à côté de ce point, mais obscurcit en plus sa propre correction, parce que Jacquette confond régulièrement l'incorporation avec la continuité et le recouvrement physiques. Toutes les interrogations de Jacquette concernant la destruction des œuvres d'art et à propos des propriétés spécifiant les cuvres d'art en tant qu'incorporées, peuvent être résolues sans difficulté - et sans soutenir, comme il cherche à le faire, que les propriétés physiques de telles œuvres d'art peuvent inclure plus qu'un certain sous-ensemble des propriétés physiques de son particulier incorporant. Il suffit de choisir le particulier incorporant avec suffisamment de précautions. En conséquence, j'ai espoir que si Jacquette n'a pas d'autres objections, lui, comme moi, trouvera dans mon ontologie des entités culturelles, « un palliatif aux limites métaphysiques du dualisme ontique, ou platonisme, et du matérialisme réducteur»; et cette libération vis-à-vis des limitations des ontologies traditionnelles ne peut être, à mon avis, que bienvenue.

Department of Philosopby, Temple University, Philadelpbia. 